\title{
RESEARCH
}

\section{Inequitable access for mentally ill patients to some medically necessary procedures}

\author{
Stephen Kisely, Mark Smith, David Lawrence, Martha Cox, Leslie Anne Campbell, Sarah Maaten
}

$\infty \quad$ See related article page 787

\section{ABSTRACT}

Background: Although universal health care aims for equity in service delivery, socioeconomic status still affects death rates from ischemic heart disease and stroke as well as access to revascularization procedures. We investigated whether psychiatric status is associated with a similar pattern of increased mortality but reduced access to procedures. We measured the associations between mental illness, death, hospital admissions and specialized or revascularization procedures for circulatory disease (including ischemic heart disease and stroke) for all patients in contact with psychiatric services and primary care across Nova Scotia.

Methods: We carried out a population-based record-linkage analysis of related data from 1995 through 2001 using an inception cohort to calculate rate ratios compared with the general public for each outcome $(n=215889)$. Data came from Nova Scotia's Mental Health Outpatient Information System, physician billings, hospital discharge abstracts and vital statistics. We estimated patients' income levels from the median incomes of their residential neighbourhoods, as determined in Canada's 1996 census.

Results: The rate ratio for death of psychiatric patients was significantly increased (1.34), even after adjusting for potential confounders, including income and comorbidity ( $95 \%$ confidence interval $[\mathrm{Cl}$ 1.29-1.40), which was reflected in the adjusted rate ratio for first admissions $(1.70,95 \% \mathrm{Cl}$ 1.67-1.72). Their chances of receiving a procedure, however, did not match this increased risk. In some cases, psychiatric patients were significantly less likely to undergo specialized or revascularization procedures, especially those who had ever been psychiatric inpatients. In the latter case, adjusted rate ratios for cardiac catheterization, percutaneous transluminal coronary angioplasty and coronary artery bypass grafts were $0.41,0.22$ and 0.34 , respectively, in spite of psychiatric inpatients' increased risk of death.

Conclusions: Psychiatric status affects survival with and access to some procedures for circulatory disease, even in a universal health care system that is free at the point of delivery. Understanding how these disparities come about and how to reduce them should be a priority for future research.

CMAJ 2007; $176(6): 779-84$
A lthough universal health care aims for equity in service delivery, socioeconomic status still affects deaths from ischemic heart disease and stroke, as well as access to specialized or revascularization procedures..$^{1,2}$ Ischemic heart disease is the major cause of death in people with psychiatric disorders. ${ }^{3,4}$ However, we do not know whether psychiatric status shows a similar pattern of increased deaths but reduced access to some services. We do know that patients under specialist psychiatric care are less likely to undergo revascularization procedures where funding of the intervention by private health insurance is an option. ${ }^{4}$ These findings may not apply when patients in primary care are included, or in countries with universal health care, such as Canada.

We therefore evaluated the association between mental illness, death, hospital admissions and specialized or revascularization procedures for patients of psychiatric services or primary care across Nova Scotia.

\section{Methods}

We used 3 administrative databases to identify anyone in contact with health services in Nova Scotia for psychiatric problems:

- The Medical Services Insurance database of all fee-forservice claims by NS physicians and psychiatrists, which included patient demographics, date of service, and diagnosis codes from the International Classification of Diseases, Ninth Revision, Clinical Modification (ICD-9-CM). ${ }^{6}$

- The Canadian Institute for Heath Information's Discharge Abstract Database, which includes hospital admissions, separation dates, diagnoses and procedures.

- Nova Scotia's Mental Health Outpatient Information System, which records service contacts, demographics and diagnoses in the public sector.

This method is consistent with the definition used by the Public Health Agency of Canada for surveillance of treated psychiatric disorders. ${ }^{7}$ We will refer to these study subjects in this article as psychiatric patients. The study protocol was approved by the Capital Health Research Ethics Board.

We included all patients whose first psychiatric contact occurred between January I, I995 and December 3I, 200I in Nova Scotia. We calculated death rates from the Statistics Canada Vital Statistics Database, using the inception cohort method. ${ }^{4}$ This reduces survivorship bias by including the entire period at 
risk. We linked data by using the provincial health card number as a unique identifier. Health card numbers were present in more than $99 \%$ of the records, irrespective of the database, and were encrypted to ensure confidentiality.

Administrative data have several advantages over community surveys or data derived from individual clinical settings. They provide accessible longitudinal data for an entire jurisdiction at relatively little cost, and both Health Canada and the Public Health Agency of Canada have used administrative data sets for chronic disease surveillance. ${ }^{7,8}$ Nevertheless, since these data were designed for billing purposes rather than disease surveillance, studies have measured accuracy over jurisdictions, over time and against other measures.

In reabstraction studies, the Discharge Abstract Database has an accuracy rate of $97 \%-99 \%$ for demographic data, including sex, type of treating physician, dates of hospital admission and discharge, and destination at discharge. ${ }^{9,10}$ In data from the Mental Health Outpatient Information System, the psychiatric diagnoses recorded showed significant agreement with the relevant items in standardized ratings completed by the treating clinician (Health of the Nation Outcome Scales). ${ }^{11}$ For instance, patients with schizophrenia and other nonaffective psychoses recorded in the Mental Health Outpatient Information System had significantly higher scores for the Health of the Nation Outcome Scales item measuring delusions and hallucinations ( $t$ test with 4 IO degrees of freedom $\left[t_{410}\right]-$ IO.2I), whereas those with depression had the highest ratings for deliberate self-harm $\left(t_{410}-4.42\right)$ and depressive symptoms $\left(t_{410}-6.32\right)$, and patients with anxiety had the highest scores for the Health of the Nation Outcome Scales item where anxiety symptoms are recorded $\left(t_{410}-3.97\right.$; all $p$ values $\left.<0.001\right)$.

Support for the validity of the Medical Services Insurance database comes from agreement across jurisdictions for the prevalance of morbidity with use of physicians' billing data. The prevalence of psychiatric disorders meeting the Public Health Agency of Canada's case definition, about $15 \%$, was similar across Nova Scotia, British Columbia, Alberta and Ontario. ${ }^{7,12}$ Hospital admissions for these disorders, as captured by the Discharge Abstract Database, accounted for $0.5 \%$ of these patients; the remaining $99.5 \%$ were determined from billings by provincial physicians. Similarly, the prevalence of diabetes, according to the expanded National Diabetes Surveillance System (a prototype for a comprehensive system of chronic physical disease surveillance, which covers complications such as circulatory disorders and death), ${ }^{8}$ was constant across Canada at about $5 \%$. The standardized mortality ratios in I999-2000 for diabetes, from data in the National Diabetes Surveillance System, were 2.Io for Nova Scotian women and I.70 for NS men, compared with ratios of 2.Io and I.90, respectively, within Canada's general population. ${ }^{8}$

Finally, although the administrative databases use ICD-9CM diagnoses, mental health clinicians make their diagnoses using the Diagnostic and Statistical Manual of Mental Disorders, 4th Edition (DSM-IV). ${ }^{13}$ Clinicians in publicly funded facilities attend DSM-IV training courses to improve their diagnostic accuracy. All DSM-IV diagnoses have equivalent ICD-9-CM codes. ${ }^{13}$

The Public Health Agency of Canada's case definition in- cludes ICD-9 diagnoses coded 290 through 319 . We also included nonspecific mental disorders outside the formal disorders covered by chapter 5 of ICD-9, such as injury of undetermined intention or psychosocial factors that influence health status, to ensure comparability with previous work ${ }^{4}$ done in Australia. Because non-chapter 5 diagnoses among patients in primary care would not always be psychiatric, we did not include them.

Where possible, we made use of codes for the ICD-9 diagnosis at hospital discharge. We used a ranking hierarchy of inpatient versus outpatient, and specialist versus primary care, which reflected both increasing percentages of patients with severe mental illness and data reliability. This approach also allowed comparison with the Australian data. ${ }^{4}$ We grouped disorders into dementia and other organic conditions (290294), psychoses (schizophrenia or nonaffective psychoses: 295, 297, 299), alcohol or drug disorders (303-305), mood disorders (affective psychoses or depression: 296, 298, 300.4, 3II), neuroses (300 except 300.4), personality disorders (30I), adjustment reactions $(308,309)$, and other mental disorders (all remaining chapter 5 and all non-chapter 5 ICD-9 diagnoses of nonspecific mental disorders).

Rates of death were measured for ischemic heart disease (ICD-9 diagnosis codes 4IO-4I4), stroke (43I-438) and other circulatory diseases (390-459). We calculated age-and-sexadjusted rates of death by direct standardization, using the average population distributions in Nova Scotia from 1995 through 200 Is the standard weights. Follow-up was judged to begin at the first contact with a clinician and end at death or on December 3I, 200I, whichever was earlier. Mortality rate ratios were calculated relative to the rate in the remaining Nova Scotian population.

We calculated standardized rates of first hospital admissions for circulatory disease in a similar way, and used these to calculate admission rate ratios relative to rates in the remaining NS population. We also compared the prevalence of specialized or revascularization procedures among patients receiving psychiatric treatment with those for the remaining general NS population using direct standardization, as previously described. We calculated first-time standardized rates and rate ratios from data from the Discharge Abstract Database for cardiac catheterization, percutaneous transluminal coronary angioplasty (PTCA), coronary artery bypass graft (CABG) or arterial implant, cerebrovascular arteriography, and carotid endarterectomy.

We used proportional hazards or logistic regression, as appropriate, to compare the risk of each outcome (death, first hospital admission or specialized procedure) from time of first contact for psychiatric disorder until the end of followup. We included principal psychiatric diagnosis, age (in 10year increments), sex, socioeconomic status, treatment setting and residence (metropolitan Halifax or elsewhere in the province). We derived income levels using the average household income from the 1996 Census for subjects' postal code at the time of initial contact, and divided them by quartile.

We assessed medical illness leading to hospital admission (as recorded in the Discharge Abstract Database) over the year before study entry by means of the modified Charlson-Deyo index. This contains I9 categories of comorbidity, primarily defined via ICD-9 codes. Each category has an associated 
weight, based on the adjusted risk of 1-year mortality. The overall score reflects the cumulative increased likelihood of 1-year mortality: the higher the score, the greater the risk. ${ }^{14}$

\section{Results}

Of the 17665 deaths from circulatory disease that occurred between I995 and 200I, 2839 were of people who had had contact with health services for psychiatric problems. The age-standardized mortality-rate ratio for psychiatric patients Was I.3I (95\% confidence interval [CI] I.25-I.36).

Moreover, mortality rate ratios were also significantly higher for the 43209 patients who had been treated in specialist services (I.80, 95\% CI I.63-I.99) compared with the I72 680 seen only in primary care (I.25, 95\% CI I.20-I.30). The difference in mortality rate ratios between the inpatients and those who had only been outpatients within the specialist group was statistically nonsignificant; likewise, the difference between men and women (Table I). Rate ratios were highest among patients with dementia (mortality rate ratio 3.07, 95\% CI 2.I9-4.29), nonaffective psychoses (3.66, 95\% CI 3.204.I9) and mood disorders (I.8I, 95\% CI 3.20-4.I9). Ischemic heart disease accounted for most of the deaths $(n=9301)$.

For first hospital admissions, the age-standardized rate ratio was I.34 (95\% CI I.32-I.37). Rate ratios were significantly higher for patients who had been treated in specialist services (I.74, 95\% CI I.68-I.8I) compared with those who had been seen only in primary care (I.29, 95\% CI I.26-I.3I). Within the specialist group, rate ratios were significantly higher in those of inpatients $(2.46,95 \% \mathrm{CI} 2.24-2.70)$. These patterns reflec- ted those for death, with the highest rate ratios found for patients with nonaffective psychoses (2.59, 95\% CI 2.39-2.80) and dementia $(2.25,95 \%$ CI I.83-2.78). Rate ratios that reflected patients' increased risk of death were similar between the sexes (Table I).

There were I3 626 specialized or revascularization procedures $(7236$ for cardiac catheterization, 2250 for PTCA, 3070 for CABG, 66I for cerebrovascular arteriography and 409 for carotid endarterectomy) performed in II 2 I 8 patients. Of these, 1685 had been psychiatric patients.

In spite of their greater risk of death, psychiatric patients were no more likely to undergo any of the 5 procedures than were the general population (Table I). Differences in procedure rates between diagnosis groups were nonsignificant except for those in dementia, where rates were between a tenth and half of those of the general population.

We used Cox or logistic regression to adjust for confounders such as age and sex (Table 2, Table 3). The risk of death from circulatory disease was 50\% higher in men than in women. As expected, increased numbers of deaths were also significantly associated with lower socioeconomic status, the presence of clinically important comorbid conditions and having had a psychiatric illness (Table 2). Rate ratios were still highest for patients with nonaffective psychoses (2.II, 95\% CI I.932.30) and dementia (I.53, 95\% CI I.4I-I.65), along with mood disorders (2.04, 95\% CI I.76-2.35). Ratios were also significantly higher for alcohol-or-drug disorders (I.5I, 95\% CI I.23I.85). Patients seen by a psychiatric specialist had more than double the risk of death (mortality rate ratio 2.42, 95\% CI I.92.9) compared with those only seen in primary care. Rates of

Table 1: Rate ratios of psychiatric patients* to members of the general population for death, initial hospital admission and medical procedure, by subject's sex

\begin{tabular}{|c|c|c|c|c|}
\hline \multirow[b]{2}{*}{ Study end point } & \multirow{2}{*}{$\begin{array}{l}\text { Diagnostic } \\
\text { codes }\end{array}$} & \multicolumn{3}{|c|}{ Unadjusted rate ratio ( $95 \%$ confidence interval) } \\
\hline & & Men & Women & All \\
\hline \multicolumn{5}{|l|}{ Death from } \\
\hline Ischemic heart disease & $410-414$ & $1.15(1.07-1.25)$ & $1.27(1.17-1.39)$ & $1.21(1.14-1.28)$ \\
\hline Stroke & $431-438$ & $1.75(1.52-2.01)$ & $1.50(1.34-1.68)$ & $1.59(1.46-1.74)$ \\
\hline Other disorders of the circulatory system & $390-459$ & $1.32(1.18-1.48)$ & $1.31(1.19-1.44)$ & $1.32(1.22-1.42)$ \\
\hline Total & & $1.28(1.21-1.35)$ & $1.33(1.26-1.41)$ & $1.31(1.25-1.36)$ \\
\hline \multicolumn{5}{|l|}{ Initial hospital admission for } \\
\hline Ischemic heart disease & $410-414$ & $1.36(1.30-1.41)$ & $1.25(1.18-1.31)$ & $1.31(1.27-1.36)$ \\
\hline Stroke & $431-438$ & $1.69(1.57-1.82)$ & $1.51(1.40-1.62)$ & $1.60(1.51-1.68)$ \\
\hline Other circulatory disease & $390-459$ & $1.41(1.37-1.45)$ & $1.25(1.22-1.29)$ & $1.32(1.30-1.35)$ \\
\hline Total & & $1.42(1.39-1.45)$ & $1.27(1.24-1.30)$ & $1.34(1.32-1.37)$ \\
\hline \multicolumn{5}{|l|}{ Medical procedure } \\
\hline Catheterization & & $1.16(0.98-1.38)$ & $0.80(0.66-0.97)$ & $1.01(0.89-1.15)$ \\
\hline Percutaneous transluminal coronary angioplasty & & $1.14(0.95-1.38)$ & $0.87(0.65-1.15)$ & $1.07(0.91-1.25)$ \\
\hline Coronary artery bypass graft & & $1.02(0.84-1.23)$ & $1.25(0.88-1.77)$ & $1.07(0.91-1.27)$ \\
\hline Cerebrovascular arteriography & & $0.63(0.39-1.00)$ & $0.82(0.49-1.35)$ & $0.73(0.51-1.05)$ \\
\hline Carotid endartectomy & & $0.82(0.47-1.43)$ & $0.92(0.55-1.54)$ & $0.59(0.58-1.27)$ \\
\hline
\end{tabular}

*Defined as anyone in contact with health services for psychiatric problems whose first psychiatric contact occurred between 1995 and 2001 , inclusive, in Nova Scotia. 
first hospital admission showed a similar pattern (Table 2).

We found several differences in patterns for specialized procedures. As before, male patients, and patients with psychiatric or comorbid medical conditions, had increased rate ratios that reflected their increased incidences of death (Table 3). Access for psychiatric patients was no better: their chance of receiving a cardiac catheterization was significantly lower than among the general population (Table 4).

Patients admitted for a psychiatric disorder were particularly disadvantaged in their chance of receiving a cardiac catheterization, PTCA or CABG - less than one-third of that of the general population (Table 4). Outpatients of the provin

Table 2: Risk factors for death and initial hospital admission

\begin{tabular}{|c|c|c|}
\hline \multirow[b]{2}{*}{ Variable } & \multicolumn{2}{|c|}{ Adjusted* risk ratio $(95 \% \mathrm{Cl})$} \\
\hline & Death & First admission \\
\hline Male & $1.50(1.46-1.55)$ & $1.16(1.15-1.19)$ \\
\hline Change per decade of age & $2.77(2.74-2.80)$ & $1.17(1.69-1.70)$ \\
\hline \multicolumn{3}{|l|}{ Income, quarterly } \\
\hline$\geq \$ 49562 \dagger$ & 1.00 & 1.00 \\
\hline$\$ 40184-\$ 49561$ & $1.26(1.19-1.32)$ & $1.06(1.04-1.08)$ \\
\hline$\$ 33776-\$ 40183$ & $1.25(1.18-1.31)$ & $1.08(1.06-1.10)$ \\
\hline$\leq \$ 33775$ & $1.34(1.27-1.41)$ & $1.12(1.10-1.14)$ \\
\hline Nonmetropolitan residence & $0.98(0.94-1.01)$ & $1.00(0.99-1.02)$ \\
\hline \multicolumn{3}{|l|}{ Comorbid disorder } \\
\hline Medical & $2.90(2.81-2.99)$ & $5.24(5.17-5.31)$ \\
\hline Psychiatric & $1.34(1.29-1.40)$ & $1.70(1.67-1.72)$ \\
\hline
\end{tabular}

*Adjusted for principal psychiatric diagnosis, age (in 10-yr increments), sex, socioeconomic status, treatment setting, residence (metropolitan Halifax or elsewhere in Nova Scotia) and medical comorbidity.

tReference category. cial mental health program were two-thirds as likely to undergo the same 3 procedures (Table 4). In a sensitivity analysis of the effect of excluding data from patients with dementia we found similar results, except for $\mathrm{CABG}$, where findings lost statistical significance. At best, therefore, rates among psychiatric patients were similar to those of the general population, in spite of the greater disease risk.

We had sufficient cases to analyze differences between individual diagnostic groups for only the 3 most frequent procedures. As expected, rates among patients with dementia were generally less than $20 \%$ of those in the general population; however, they were significantly lower as well among those with nonaffective psychoses for therapeutic cardiac catheterization (0.46, 95\% CI 0.29-0.73), PTCA (0.46, 95\% CI $0.29-0.73$ ) and CABG (0.24, 95\% CI 0.10-0.58).

In contrast to their increased risk, subjects in the lowestincome quarter were also significantly less likely to receive 3 of the 5 procedures: cardiac catheterization, PTCA and CABG (Table 4). Although their rate of death was no lower than those from metropolitan Halifax, patients from elsewhere in the province were significantly less likely to undergo catheterization, PTCA or cerebrovascular arteriography (Table 4).

\section{Interpretation}

As family physicians provide most psychiatric treatment, our findings give a comprehensive picture of the relationship between psychiatric disorder and circulatory disease. They also allow a comparison between health systems with and without universal health coverage, such as those in Canada and Australia, respectively. Results were very similar. ${ }^{4}$ Although rates of first hospital admission reflected the increased risk of death among psychiatric patients, the pattern was different for interventions more likely to be elective in nature. Here, the possibility of specialized procedures for circulatory disorders

Table 3: Risk factors for selected specialist procedures, adjusted for principal psychiatric diagnosis, age (in 10-year increments), sex, socioeconomic status, treatment setting, residence (metropolitan Halifax or elsewhere in Nova Scotia) and medical comorbidity

Adjusted rate ratios $(95 \%$ confidence interval)

\begin{tabular}{|c|c|c|c|c|c|}
\hline \multirow[b]{2}{*}{ Variable } & \\
\hline & Catheterization & PTCA & $\begin{array}{c}\text { Coronary artery } \\
\text { bypass graft }\end{array}$ & $\begin{array}{c}\text { Carotid } \\
\text { endartectomy }\end{array}$ & $\begin{array}{c}\text { Cerebrovascular } \\
\text { arteriography }\end{array}$ \\
\hline Male & $1.53(1.46-1.61)$ & $2.35(2.15-2.57)$ & $3.31(3.05-3.59)$ & $1.50(1.23-1.83)$ & $0.86(0.74-1.00)$ \\
\hline \multicolumn{6}{|l|}{ Income, quarterly } \\
\hline$\geq \$ 49562^{*}$ & 1.00 & 1.00 & 1.00 & 1.00 & 1.00 \\
\hline$\$ 33776-\$ 40183$ & $0.98(0.91-1.06)$ & $0.90(0.79-1.02)$ & $0.90(0.81-1.01)$ & $1.13(0.82-1.56)$ & $1.13(0.91-1.41)$ \\
\hline$\leq \$ 33775$ & $0.84(0.78-0.91)$ & $0.74(0.65-0.84)$ & $0.83(0.74-0.92)$ & $1.05(0.76-1.44)$ & $0.87(0.70-1.10)$ \\
\hline Nonmetropolitan residence & $0.75(0.71-0.79)$ & $0.74(0.68-0.82)$ & $0.92(0.85-1.00)$ & $0.95(0.77-1.18)$ & $0.53(0.45-0.63)$ \\
\hline \multicolumn{6}{|l|}{ Comorbid disorder } \\
\hline Medical & $1.06(1.01-1.11)$ & $1.10(1.01-1.21)$ & $1.10(1.02-1.19)$ & $2.44(1.98-2.99)$ & $3.02(2.57-3.55)$ \\
\hline
\end{tabular}

Note: PTCA = percutaneous transluminal coronary angioplasty.

*Reference category. 
Table 4: Risk factors for selected specialist procedures, by psychiatric setting

\begin{tabular}{|c|c|c|c|c|}
\hline \multirow[b]{2}{*}{ Variable } & \multicolumn{4}{|c|}{ Adjusted rate ratio* ( $95 \%$ confidence interval) } \\
\hline & Catheterization & & PTCA & CABG \\
\hline \multicolumn{5}{|l|}{ Specialist services } \\
\hline Hospital inpatients & $0.41(0.26-0.65)$ & 0.22 & $(0.07-0.69)$ & $0.34(0.15-0.77)$ \\
\hline Outpatients $†$ & $0.56(0.44-0.70)$ & 0.59 & $(0.39-0.89)$ & $0.66(0.47-0.93)$ \\
\hline Private specialists & $0.88(0.61-1.27)$ & 0.96 & $(0.53-1.76)$ & $0.71(0.39-1.29)$ \\
\hline Primary care & $0.99(0.93-1.07)$ & 1.05 & $(0.93-1.19)$ & $0.98(0.88-1.01)$ \\
\hline
\end{tabular}

Note: PTCA = percutaneous transluminal coronary angioplasty, CABG = coronary artery bypass graft *Of patients who used psychiatric services during the study period compared with members of the general population, adjusted for for principal psychiatric diagnosis, age (in 10-yr increments), sex, socioeconomic status, treatment setting, residence (metropolitan Halifax or elsewhere in Nova

Scotia) and medical comorbidity.

†Data from Nova Scotia's Mental Health Outpatient Information System.

was found to be reduced in both countries. Other factors associated with increased rates of death but reduced access to specialized procedures were low income and rural residence.

Lifestyle, such as alcohol or tobacco use, and side effects of a psychotropic medication may contribute to increased numbers of deaths among psychiatric patients. ${ }^{3,15-26}$ Our current study and other work from Australia suggest another factor: the disparity in rates of specialized or revascularization procedures between those with psychiatric disorders and the general population, especially for those admitted to hospital for a psychiatric disorder. Outpatients in the provincial mental health program were also significantly less likely to receive several of the procedures, although the effect was less marked than among those who had been inpatients. Lower rates of private health coverage among psychiatric patients may affect access to specialized or revascularization procedures in Australia. Only $13 \%$ of Australians with psychotic disorders have private health insurance, compared with $32 \%$ of the general population. ${ }^{4} \mathrm{We}$ now know that the same pattern of access occurs in Canada's universal health system, even after adjustments for income.

This inequity is consistent with findings from Ontario, where studies ${ }^{1,2,27}$ have shown that other marginalized populations, such as those with a low annual income, are less likely to receive specialized procedures. Ability to pay is clearly not the sole determinant. Is this discrepancy also caused by problems in registering with a family physician, missed appointments, or difficulties in communication or in scheduling appointments?

This study had several limitations. Administrative data are subject to recording bias, especially for diagnosis; to minimize this, we emphasized overall psychiatric illness rather than subcategories. Subdividing psychiatric illness by treatment setting (such as inpatient, ambulatory or primary care) is also less subject to recording bias. We did not have data on patients' marital status, duration of contact with services or educational level. Neither were we able to control for alcohol or tobacco use. This would not, however, explain our finding of increased risk of death and first hospital admission in the presence of a reduced likelihood of specialized procedures.

Administrative data lack indicators of circulatory disease severity, such as level of consciousness and disability. Although we could not consider legal status, other studies have shown no significant differences in rates of death from any cause between involuntary and voluntary patients. ${ }^{4,15}$ Moreover, we were able to adjust for other predictors, including age, sex, socioeconomic status and comorbid illness. Nevertheless, our additive model did not adjust for all possible confounders or interactions. Our results on the effect of income or rural residency apply to people in contact with psychiatric services, and may not generalize to other populations. Small numbers impeded multivariable analyses of less common diagnoses or procedures.

In conclusion, psychiatric status affects access to some procedures for circulatory disease and risk of death, even in a universal health care system that is free at the point of delivery. A greater understanding of the reasons for this would help to reduce these disparities.

\section{This article has been peer reviewed.}

From the Departments of Psychiatry, Community Health and Epidemiology (Kisely) and the Population Health Research Unit (Smith, Cox), Dalhousie University, and the Health Outcomes Unit (Campbell), Capital District Health Authority, Halifax, NS; the Centre for Developmental Health (Lawrence), Curtin University of Technology, Perth, Australia; and the Institute for Clinical Evaluative Sciences (Maaten), Toronto, Ont.

\section{Competing interests: None declared.}

Contributors: Stephen Kisely had the original study concept, wrote the first draft of the report and assisted with data analysis. Martha Cox extracted the data and carried out the multivariate analyses. Sarah Maaten wrote the program to create the regression models. Leslie Anne Campbell assisted with the interpretation of the findings. David Lawrence contributed to the design of the study and assisted Martha Cox with the calculation of mortality ratios. All of these authors reviewed and revised the report for content. Mark Smith wrote the software program to identify and extract cases. All authors approved the final version for publication.

Acknowledgements: This study was supported by a Grant-in-Aid award (2004-05) from the Heart and Stroke Foundation of Nova Scotia. The data used in this report were made available by the Population Health Research Unit within Dalhousie University's Department of Community Health and Epidemiology. The Population Health Research Unit is a university-based research and support group that conducts systematic research into population health, health services and their inter-relationships. The Province of Nova Scotia supplies the unit with complete Medicare and hospital files suitable for research purposes. The unit also has access to a variety of other data sources including clinical databases and large scale population surveys. Although this research is based on data obtained from the unit, the observations and opinions expressed are those of the authors and do not represent those of the Population Health Research Unit. 


\section{REFERENCES}

I. Alter DA, Naylor CD, Austin P, et al. Effects of socioeconomic status on access to invasive cardiac procedures and on mortality after acute myocardial infarction. $N$ Engl J Med 1999;341:1359-67.

2. Kapral MK, Wang H, Mamdani M, et al. Effect of socioeconomic status on treatment and mortality after stroke. Stroke 2002;33:268-73.

3. Harris EC, Barraclough B. Excess mortality of mental disorder. Br J Psychiatry I998;173:II-53.

4. Lawrence DM, Holman CD, Jablensky AV, et al. Death rate from ischaemic heart disease in Western Australian psychiatric patients 1980-1998. BrJ Psychiatry 2003; I82:3I-6.

5. Population Health Research Unit, Faculty of Medicine, Dalhousie University. Research data repository. Available: http://metadata.phru.dal.ca (accessed 2007 Jan 25).

6. World Health Organization. Mental disorders in primary care: glossary and guide to their classification in accordance with the ninth revision of the International Classification of Diseases. Geneva: The Organization; 1978.

7. Gilbert C, Jones W, Schopflocher D, et al. Use of provincial administrative data for surveillance of mental disorders: feasibility study. Ottawa: Surveillance Division, Centre for Chronic Disease Prevention and Control, Public Health Agency of Canada; 2007. In press.

8. National Diabetes Surveillance System. Responding to the challenge of diabetes in Canada: first report of the National Diabetes Surveillance System (NDSS). Ottawa: Her Majesty the Queen in Right of Canada, represented by the Minister of Health; 2003. Available: www.phac-aspc.gc.ca/ccdpc-cpcmc/ndss-snsd/english/pubs _reports/pdf/WEB_NDSS_English_Report-nocover.pdf (accessed 2007 Jan 25).

9. Ontario Hospital Association; Hospital Medical Records Institute, Ontario Ministry of Health. Report of the Ontario Data Quality Reabstracting Study. Toronto: The Association; I99I.

Io. Williams JI, Young W. Inventory of studies on the accuracy of Canadian health administrative databases. Toronto: Institute for Clinical Evaluative Sciences; 1996.

II. Kisely S, Campbell L, Crossman D, et al. Are the health of the nation outcome scales a valid and practical instrument to measure outcomes in North America? A three-site evaluation across Nova Scotia. Community Ment Health J DOI: I0.I007/ sio597-006-9067-2. Epub 2006 Oct 5 ahead of print.

I2. Gilbert C. Mental health surveillance (slides). Proceedings of the Mental Health Research Showcase, 2006 Oct 30-Nov I, Banff (AB). Edmonton: Alberta Mental
Health Board; 2006. Available: www.buksa.com/showcase/docs/o2oD-Gilbert.pdf (accessed 2007 Jan 26).

13. American Psychiatric Association. Diagnostic and statistical manual of mental disorders. $4^{\text {th }}$ ed. Washington: American Psychiatric Association; I994.

14. Deyo RA, Cherkin DC, Ciol MA. Adapting a clinical comorbidity index for use with ICD-9-CM administrative databases. J Clin Epidemiol 1992;45:613-9.

I5. Crisanti AS, Love EJ. Mortality among involuntarily admitted psychiatric patients: a survival analysis. Soc Psychiatry Psychiatr Epidemiol 1999;34:627-33.

I6. Black DW. Iowa record-linkage study: death rates in psychiatric patients. $J$ Affect Disord I998;50:277-82.

17. Brown S, Inskip H, Barraclough B. Causes of the excess mortality of schizophrenia. BrJPsychiatry 2000;177:212-7.

I8. Lambert TJR, Velakoulis S, Pantelis C. Medical comorbidity in schizophrenia. Med JAust 2003;178:S67-70.

19. Kisely S, Wise M, Preston N, et al. A group intervention to reduce smoking in individuals with psychiatric disorder: brief report of a pilot study. Aust J Public Health 2003;27:6I-3.

20. Cuijpers P, Smit F. Excess mortality in depression: a meta-analysis of community studies. JAffect Disord 2002;72:227-36.

2I. McCreadie R, Macdonald E, Blacklock C, et al. Dietary intake of schizophrenia patients in Nithsdale, Scotland: case-control study. BMJ I998;317:784-5.

22. Elming H, Sonne J, Lublin HK. The importance of the QT interval: a review of the literature. Acta Psychiatr Scand 2003;107:96-Ior.

23. Reilly JG, Ayis SA, Ferrier IN, et al. Thioridazine and sudden unexplained death in psychiatric inpatients. BrJ Psychiatry 2002;180:515-22.

24. Vanina Y, Podolskaya A, Sedky K, et al. Body weight changes associated with psychopharmacology. Psychiatr Serv 2002;53:842-7.

25. Cohen M, Dembling B, Schorling J. The association between schizophrenia and cancer: a population-based mortality study. Schizophr Res 2002;57:139-46.

26. Craig TJ, Lin SP. Death and deinstitutionalization. Am J Psychiatry I981;138:224-7.

27. Huang J, Zhou S, Groome P, et al. Factors affecting the use of palliative radiotherapy in Ontario. J Clin Oncol 2001;19:137-44.

Correspondence to: Dr. Stephen Kisely, Centre for Clinical

Research, 5790 University Ave., Rm. 425, Halifax, $\mathrm{NS}_{3} \mathrm{H}_{\mathrm{I}} \mathrm{V}_{7}$;

fax 902 494-I597; stephen.kisely@cdha.nshealth.ca

\section{How you can get involved in the CMA!}

The CMA is committed to providing leadership for physicians and promoting the highest standard of health and health care for Canadians. To strengthen the Association and be truly representative of all Canadian physicians, the CMA needs to hear from members interested in serving in elected positions and on appointed committees and advisory groups.

The CMA structure comprises both governing bodies and advisory bodies either elected by General Council or appointed by the CMA Board of Directors. The Board of Directors - elected by General Council — has divisional, affiliate, resident and student representation, is responsible for the overall operation of the CMA and reports to General Council on issues of governance. CMA councils and committees advise the Board of Directors and make recommendations on specific issues of concern to physicians and the public. Five core councils and committees consist of either divisional or regional representation, while other statutory and special committees, and task forces consist of individuals with interest and expertise in subject-specific fields. Positions on one or more of these committees may become available in the coming year.

For further information on how you can get involved, please contact:

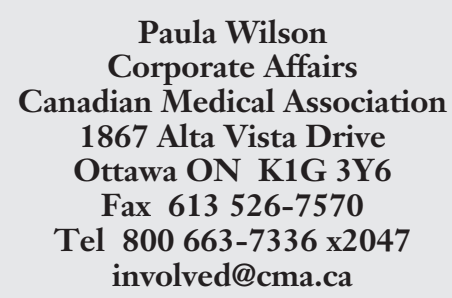

By getting involved, you will have an opportunity to make a difference.

We hope to hear from you!

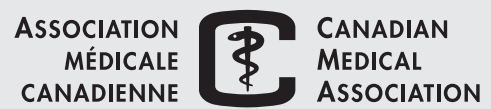

